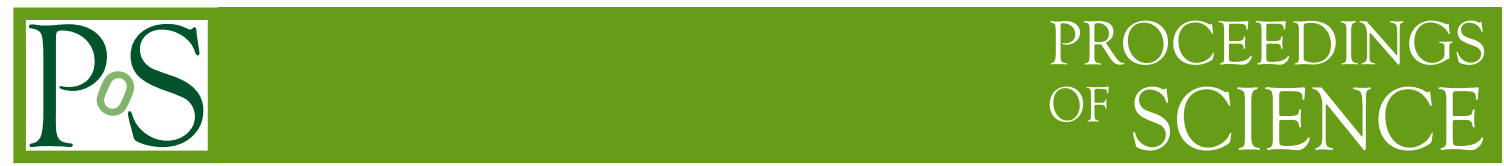

\title{
Calculation of Multi-Loop Integrals with SecDec-3.0
}

\author{
Johannes Schlenk*, Tom Zirke \\ Max Planck Institute for Physics, Föhringer Ring 6, 80805 Munich, Germany \\ E-mail: jschlenk@mpp.mpg.de, zirke@mpp.mpg.de
}

In this contribution we discuss new features of SecDec-3.0, a public program for the evaluation of dimensionally-regulated parametric integrals using sector decomposition. We will focus on two main aspects: the implementation of an improved geometric decomposition algorithm and the recently added support for complex masses.

12th International Symposium on Radiative Corrections (Radcor 2015) and LoopFest XIV (Radiative Corrections for the LHC and Future Colliders)

15-19 June 2015

UCLA Department of Physics \& Astronomy Los Angeles, CA, USA

${ }^{*}$ Speaker. 


\section{Introduction}

As Run II of the LHC at $13 \mathrm{TeV}$ is progressing and statistical uncertainties are decreasing, precise predictions at higher loop orders involving many scales are needed to push the precision frontier. An important building block of higher order predictions is the calculation of master integrals, which, however, often cannot be performed analytically with current technology.

An alternative is offered by numerical methods, such as sector decomposition [1-4], which handles the $\varepsilon$-expansion of dimensionally regulated multi-loop Feynman integrals and their numerical calculation. The public program SECDEC [5-7], whose latest features we shall discuss in this paper, provides an automated implementation of this algorithm. Other publicly available codes are sector_decomposition [8] and Fiesta [9-12].

The main part of this paper is structured as follows: We begin with a recap of the sector decomposition algorithm in Section 2. Section 3 is dedicated to the new features of SECDEC 3.0, with emphasis on the geometric decomposition strategy and the implementation of complex masses. Finally we give our conclusion in Section 4.

\section{Sector Decomposition}

Although the sector decomposition algorithm is applicable to a more general class of parametric integrals, let us consider as a starting point of our discussion the Feynman parametrization of an $L$-loop integral,

$$
I=\frac{(-1)^{N_{v}}}{\prod_{j=1}^{N} \Gamma\left(v_{j}\right)} \Gamma\left(N_{v}-L D / 2\right) \int_{0}^{\infty} \prod_{j=1}^{N} \frac{\mathrm{d} x_{j}}{x_{j}} x_{j}^{v_{j}} \delta\left(1-\sum_{l=1}^{N} x_{l}\right) \mathcal{N} \frac{\mathcal{U}^{N_{v}-(L+1) D / 2}}{\mathcal{F}^{N_{v}-L D / 2}},
$$

where $\mathcal{U}$ and $\mathcal{F}$ are the graph polynomials, $\mathcal{N}$ is a numerator appearing in tensor integrals, and $D=4-2 \varepsilon$. The parameters $v_{j}$ denote the propagator exponents and $N_{v}=\sum_{j=1}^{N} v_{j}$.

The basic idea of the algorithm is to split up the integration region into multiple parts with non-overlapping singularities and perform a change of variables to map to the unit hypercube afterwards. There exist several different decomposition algorithms, which will be discussed in more detail in Section 3.1. As a result the singularities are isolated as factors of the form $x^{-a+b \varepsilon}$ with $a>0$ so that they can be subtracted systematically. This makes it possible to perform an expansion in $\varepsilon$. Finally, the coefficients to each order in $\varepsilon$ can be calculated numerically.

\section{New Features of SecDec-3.0}

The latest version of the program SECDEC can be obtained from hepforge on the url:

$$
\text { http://secdec.hepforge.org/ }
$$

It requires a working version of Perl and Mathematica version 7 or higher, as well as a C++ compiler.

Users familiar with earlier versions of the program will notice the improved user interface. It was designed with mainly two goals in mind: firstly to facilitate interfacing with reduction programs, e.g. by implementing support of zero and negative propagator exponents $v_{j}$, and secondly to 
simplify scans over ranges of kinematical parameters, which can now be done by simply providing a list of phase space points in the kinem. input file.

On the technical side, the list of available numerical integrators, so far consisting of Cuba [13, 14] and Bases [15], has been extended by Cquad [16] and the NIntegrate routine from Mathematica. Furthermore the compilation and numerical integration can now be parallelized in a straightforward way on a cluster, where the submission systems condor and PBS are supported.

Also the field of possible applications was enlarged: Support for integrals with linear propagators following the Feynman $+i \delta$ prescription has been implemented, as well as support for complex masses. The latter will be discussed in Section 3.2. In addition, general parametric integrals may now contain $\varepsilon$-dependent dummy functions.

Another major new feature, which shall be presented in the following, is the implementation of a geometric decomposition algorithm.

\subsection{Geometric Decomposition Algorithm}

The heuristic decomposition algorithm implemented in SECDEC suffers from the problem that in some cases an infinite recursion can occur and no full decomposition is obtained. The algorithms described in Refs. [8] and [9] are guaranteed to terminate, but generically produce a larger number of sectors. A different approach based on convex geometry was introduced by Kaneko and Ueda in Refs. $[17,18]$. This algorithm is guaranteed to terminate, while also keeping the number of sectors small.

In addition to the heuristic algorithm, the most recent version of SECDEC implements the original algorithm of Kaneko and Ueda (G1) and an improved geometric decomposition algorithm (G2). Here we describe the improved geometric decomposition algorithm G2.

In contrast to other sector decomposition algorithms where a primary sector decomposition is performed, the Cheng-Wu theorem [19,20] is used to integrate out the Dirac delta in Eq. (2.1). This amounts to replacing the $\delta$-distribution by $\delta\left(1-x_{N}\right)$.

In the next step the Newton polytope $\Delta$ of the polynomial $\mathcal{U} \cdot \mathcal{F} \cdot \mathcal{N}=\sum_{j=1}^{m} c_{j} \mathbf{x}^{\mathbf{v}_{j}}$ is calculated, which is defined as the convex hull of the $(N-1)$-dimensional exponent vectors $\mathbf{v}_{j}$ :

$$
\Delta=\operatorname{ConvHull}\left(\mathbf{v}_{1}, \ldots, \mathbf{v}_{m}\right) .
$$

Here the multi-index notation $\mathbf{x}^{\mathbf{v}_{j}}=\prod_{i} x_{i}^{\left(\mathbf{v}_{j}\right)_{i}}$ is used. The polytope $\Delta$ contains all necessary information for the sector decomposition. Due to the Minkowski-Weyl theorem there exists a second representation of the polytope $\Delta$ as an intersection of halfspaces defined by the facet normal vectors $\mathbf{n}_{F}[21]:$

$$
\Delta=\bigcap_{F}\left\{\mathbf{x} \in \mathbb{R}^{N-1} \mid\left\langle\mathbf{x}, \mathbf{n}_{F}\right\rangle+a_{F} \geq 0\right\} .
$$

In SECDEC the program Normaliz 2.10.1 $[22,23]$ is used to translate between vertex and facet representations, Eq. (3.1) and Eq. (3.2), respectively.

For each extremal vertex of $\Delta$ indexed by the parameter $j$, a sector is introduced. The sector is bounded by the facet vectors $\mathbf{n}_{F}$ incident to the vertex $j$. In order to map the integration region back to the unit hypercube, the local change of variables

$$
x_{i}=\prod_{F \in S_{j}} y_{F}^{\left\langle\mathbf{e}_{i}, \mathbf{n}_{F}\right\rangle}
$$


is performed in sector $j$. The vectors $\mathbf{e}_{i}$ denote the orthonormal basis of $\mathbb{R}^{N-1}$, the set $S_{j}$ contains the facets incident to the vertex $j$. In cases where the set $S_{j}$ contains more than $N-1$ elements, an additional triangulation of the sector is needed. In SECDEC the triangulation algorithm implemented in NORMALIZ is used for this purpose.

Compared to the other strategies implemented in SECDEC, strategy G2 is the fastest method and it usually produces the smallest number of sectors.

As an example we decompose the two-loop vacuum integral with one massive and two massless propagators using strategy G2. After employing the Cheng-Wu theorem to integrate out the massive Feynman parameter $x_{3}$, the Feynman integral becomes

$$
I=\overbrace{\because \ldots, \ldots}^{m}=-\Gamma(-1+2 \varepsilon)\left(m^{2}\right)^{1-2 \varepsilon} \int_{0}^{\infty} \frac{\mathrm{d} x_{1} \mathrm{~d} x_{2}}{\left(x_{1}^{1} x_{2}^{0}+x_{1}^{1} x_{2}^{1}+x_{1}^{0} x_{2}^{1}\right)^{2-\varepsilon}}
$$

The exponent vectors

$$
\mathbf{v}_{1}=\left(\begin{array}{l}
1 \\
0
\end{array}\right), \mathbf{v}_{2}=\left(\begin{array}{l}
1 \\
1
\end{array}\right), \mathbf{v}_{3}=\left(\begin{array}{l}
0 \\
1
\end{array}\right)
$$

can be read off from the polynomial in the denominator of Eq. (3.4) and the associated Newton polytope $\Delta$ is shown in Fig. 1.

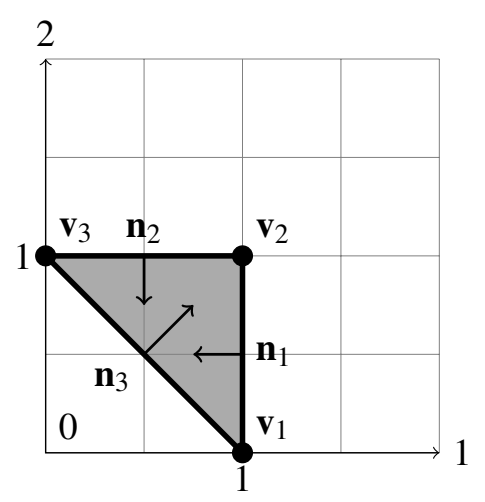

Figure 1: Newton polytope $\Delta$ associated to the two loop vacuum integral of Eq. (3.4)

The facet normal vectors

$$
\begin{aligned}
& \mathbf{n}_{1}=\left(\begin{array}{c}
-1 \\
0
\end{array}\right) \mathbf{n}_{2}=\left(\begin{array}{c}
0 \\
-1
\end{array}\right) \mathbf{n}_{3}=\left(\begin{array}{l}
1 \\
1
\end{array}\right) \\
& a_{1}=1 \quad a_{2}=1 \quad a_{3}=-1
\end{aligned}
$$

together with Eq. (3.2) specify the facet representation of the polytope $\Delta$. The sets $S_{j}$ associated to the three extremal vertices $\mathbf{v}_{1}$ to $\mathbf{v}_{3}$ are $S_{1}=\{3,1\}, S_{2}=\{1,2\}$ and $S_{3}=\{2,3\}$. In this case no additional triangulation is necessary since the size of the sets already equals $N-1$. The change of variables defined in Eq. (3.3) can then be written as

$$
\begin{gathered}
x_{1}=y_{1}^{-1} y_{3}, \\
x_{2}=y_{2}^{-1} y_{3}
\end{gathered}
$$


leading to the decomposed form of the vacuum integral

$$
I=-\Gamma(-1+2 \varepsilon)\left(m^{2}\right)^{1-2 \varepsilon} \int_{0}^{1} \mathrm{~d} y_{1} \mathrm{~d} y_{2} \mathrm{~d} y_{3} \frac{y_{1}^{-\varepsilon} y_{2}^{-\varepsilon} y_{3}^{-1+\varepsilon}}{\left(y_{1}+y_{2}+y_{3}\right)^{2-\varepsilon}}\left[\boldsymbol{\delta}\left(1-y_{2}\right)+\delta\left(1-y_{3}\right)+\delta\left(1-y_{1}\right)\right],
$$

where the $\delta$-distributions correspond to the sets $S_{1}$ to $S_{3}$.

\subsection{Complex Masses}

In certain applications, especially in the electroweak context, the width of unstable particles can be important. A consistent treatment is provided by the complex-mass scheme [24,25], where the width $\Gamma$ is included as a negative imaginary part of the mass via the replacement

$$
m^{2} \rightarrow m_{c}^{2} \equiv m^{2}\left(1-i \frac{\Gamma}{m}\right)
$$

The graph polynomial $\mathcal{F}$ then has the form

$$
\mathcal{F}=\mathcal{F}_{0}+\mathcal{U} \sum_{j} x_{j}\left(m_{j}^{2}-i m_{j} \Gamma_{j}\right),
$$

i.e. the widths induce a negative imaginary part:

$$
\operatorname{Im} \mathcal{F}=-\mathcal{U} \sum_{j} x_{j} m_{j} \Gamma_{j}
$$

In general, for zero widths, $\mathcal{F}$ will exhibit kinematic-dependent zeros even after sector decomposition, which can be avoided by a suitable deformation of the integration contour [26-28]. Similarly, a non-zero width can help to avoid these singular regions as well, but one cannot expect this to lead to a stable numerical integration in all cases. Thus it makes sense to try to combine the two in a consistent way, which should be possible since both the contour deformation and the complex masses are required to produce only negative imaginary parts in order to fulfill the Feynman $+i \delta$ prescription. For SECDEC-3.0 we have chosen

$$
\begin{aligned}
\vec{z}(\vec{x}) & =\vec{x}-i \vec{\tau}(\vec{x}), \\
\tau_{k} & =\lambda x_{k}\left(1-x_{k}\right) \frac{\partial \operatorname{Re} \mathcal{F}}{\partial x_{k}},
\end{aligned}
$$

i.e. to set the widths to zero in the definition of the deformation. For small deformations we then have

$$
\begin{aligned}
\mathcal{F}(\vec{z}(\vec{x}))= & \operatorname{Re} \mathcal{F}(\vec{x})+i \operatorname{Im} \mathcal{F}(\vec{x})-i \lambda \sum_{k} x_{k}\left(1-x_{k}\right)\left[\left(\frac{\partial \operatorname{Re} \mathcal{F}}{\partial x_{k}}\right)^{2}+i \frac{\partial \operatorname{Re} \mathcal{F}}{\partial x_{k}} \frac{\partial \operatorname{Im} \mathcal{F}}{\partial x_{k}}\right] \\
& -\frac{\lambda^{2}}{2} \sum_{k, l} x_{k}\left(1-x_{k}\right) x_{l}\left(1-x_{l}\right) \frac{\partial \operatorname{Re} \mathcal{F}}{\partial x_{k}} \frac{\partial \operatorname{Re} \mathcal{F}}{\partial x_{l}}\left[\frac{\partial^{2} \operatorname{Re} \mathcal{F}}{\partial x_{k} \partial x_{l}}+i \frac{\partial^{2} \operatorname{Im} \mathcal{F}}{\partial x_{k} \partial x_{l}}\right]+\mathcal{O}\left(\lambda^{3}\right) .
\end{aligned}
$$

Up to order $\lambda$, the imaginary parts induced by the widths and the contour deformation are both negative as they should. The term involving $\frac{\partial \operatorname{Im} \mathcal{F}}{\partial x_{k}}$ does no harm because it is purely real. At order $\lambda^{2}$, however, $\operatorname{Im} \mathcal{F}$ leads to an imaginary part of indefinite sign, which would otherwise have been 
the case at one order higher in $\lambda$. On the other hand, this term is proportional to $\frac{\Gamma_{j}}{m_{j}}$ and thus suppressed since the widths should be small compared to the corresponding masses. Therefore we conclude that for a sufficiently small value of $\lambda$, one can consistently combine complex masses and contour deformation.

The support for complex masses is included in SECDEC from version 3.0.8 and can be enabled by setting complexmasses=1 in the param. input file. It may be used with and without contour deformation. If complex masses are switched on, the kinem. input file expects two numbers for each mass parameter:

$$
\cdots \operatorname{Re} m_{c, 1}^{2} \operatorname{Im} m_{c, 1}^{2} \operatorname{Re} m_{c, 2}^{2} \operatorname{Im} m_{c, 2}^{2} \cdots
$$

As an example the calculation of a one-loop pentagon with complex masses can be found in the loop/demos/11_complexmass folder.

\section{Conclusions}

The new version 3.0 of the program SECDEC comes with an improved user interface and opens up several new possibilities: Two geometric decomposition strategies, which are guaranteed to stop, have been implemented, as well as support for linear propagators, complex masses, $\varepsilon$-dependent dummy functions, and new integration routines. Furthermore, the more flexible definition of integrals facilitates the link to reduction programs. There is an option to scan over phase-space points, and the new cluster mode makes it easier to perform calculations on modern super computers.

With these features, SECDEC is now ready for the application to phenomenologically relevant processes and has the potential to become an important building block in a future automated twoloop setup.

\section{Acknowledgments}

We would like to thank the members of the SECDEC team, Sophia Borowka, Gudrun Heinrich, Stephan Jahn, Stephen Jones, and Matthias Kerner for the successful collaboration and helpful comments on the manuscript.

\section{References}

[1] K. Hepp, Proof of the Bogolyubov-Parasiuk theorem on renormalization, Commun. Math. Phys. 2 (1966) 301-326.

[2] M. Roth and A. Denner, High-energy approximation of one-loop Feynman integrals, Nucl. Phys. B479 (1996) 495-514, [hep-ph/ 9605420$]$.

[3] T. Binoth and G. Heinrich, An automatized algorithm to compute infrared divergent multi-loop integrals, Nucl. Phys. $\mathbf{B 5 8 5}$ (2000) 741-759, [hep-ph / 0004013 ].

[4] G. Heinrich, Sector Decomposition, Int. J. Mod. Phys. A23 (2008) 1457-1486, [arXiv:0803.4177].

[5] J. Carter and G. Heinrich, SecDec: A general program for sector decomposition, Comput.Phys.Commun. 182 (2011) 1566-1581, [arXiv: 1011.5493 ]. 
[6] S. Borowka, J. Carter, and G. Heinrich, Numerical Evaluation of Multi-Loop Integrals for Arbitrary Kinematics with SecDec 2.0, Comput.Phys.Commun. 184 (2013) 396-408, [arXiv: 1204.4152 ].

[7] S. Borowka, G. Heinrich, S. P. Jones, M. Kerner, J. Schlenk, and T. Zirke, SecDec-3.0: numerical evaluation of multi-scale integrals beyond one loop, Comput. Phys. Commun. 196 (2015) 470-491, [arXiv:1502.0659].

[8] C. Bogner and S. Weinzierl, Resolution of singularities for multi-loop integrals, Comput.Phys.Commun. 178 (2008) 596-610, [arXiv: 0709.4092 ].

[9] A. Smirnov and M. Tentyukov, Feynman Integral Evaluation by a Sector decomposiTion Approach (FIESTA), Comput.Phys.Commun. 180 (2009) 735-746, [arXiv : 0807 . 4129].

[10] A. Smirnov, V. Smirnov, and M. Tentyukov, FIESTA 2: Parallelizeable multiloop numerical calculations, Comput.Phys.Commun. 182 (2011) 790-803, [arXiv:0912.0158].

[11] A. V. Smirnov, FIESTA 3: cluster-parallelizable multiloop numerical calculations in physical regions, Comput.Phys.Commun. 185 (2014) 2090-2100, [arXiv: 1312.3186].

[12] A. V. Smirnov, FIESTA 4: optimized Feynman integral calculations with GPU support, arXiv:1511.0361.

[13] T. Hahn, CUBA: A library for multidimensional numerical integration, Comput. Phys. Commun. 168 (2005) 78-95, [hep-ph/ 0404043$].$

[14] T. Hahn, Concurrent Cuba, J. Phys. Conf. Ser. 608 (2015), no. 1 012066, [arXiv: 1408 . 6373].

[15] S. Kawabata, A New version of the multidimensional integration and event generation package BASES/SPRING, Comp. Phys. Commun. 88 (1995) 309-326.

[16] P. Gonnet, Increasing the reliability of adaptive quadrature using explicit interpolants, CoRR abs/1006.3962 (2010).

[17] T. Kaneko and T. Ueda, A Geometric method of sector decomposition, Comput.Phys.Commun. 181 (2010) 1352-1361, [arXiv:0908.2897].

[18] T. Kaneko and T. Ueda, Sector Decomposition Via Computational Geometry, PoS ACAT2010 (2010) 082, [arXiv:1004.5490].

[19] H. Cheng and T. Wu, Expanding Protons: Scattering at High Energies. The MIT Press, 1987.

[20] V. A. Smirnov, Feynman integral calculus. Springer, 2006.

[21] T. Oda, Convex bodies and algebraic geometry. Springer, 1988.

[22] W. Bruns, B. Ichim, and C. Söger, The power of pyramid decomposition in Normaliz, ArXiv e-prints (June, 2012) [arXiv:1206.1916].

[23] W. Bruns, B. Ichim, T. Römer, and C. Söger, "Normaliz. Algorithms for rational cones and affine monoids. Available from http://www.math.uos.de/normaliz."

[24] A. Denner, S. Dittmaier, M. Roth, and D. Wackeroth, Predictions for all processes $e^{+} e^{-} \rightarrow 4$ fermions $+\gamma$, Nucl. Phys. B560 (1999) 33-65, [hep-ph/9904472].

[25] A. Denner, S. Dittmaier, M. Roth, and L. H. Wieders, Electroweak corrections to charged-current $e^{+} e^{-} \rightarrow 4$ fermion processes: Technical details and further results, Nucl. Phys. B724 (2005) 247-294, [hep-ph/ 05050 42]. [Erratum: Nucl. Phys.B854,504(2012)].

[26] D. E. Soper, Techniques for QCD calculations by numerical integration, Phys. Rev. D62 (2000) 014009, [hep-ph/9910292]. 
[27] Z. Nagy and D. E. Soper, Numerical integration of one-loop Feynman diagrams for N-photon amplitudes, Phys. Rev. D74 (2006) 093006, [hep-ph/ 0610028 ].

[28] T. Binoth, J. P. Guillet, G. Heinrich, E. Pilon, and C. Schubert, An algebraic / numerical formalism for one-loop multi-leg amplitudes, JHEP 10 (2005) 015, [hep-ph/ 0504267 ]. 